\title{
Higher Order Terms of Kerr Parameter for Blandford-Znajek Monopole Solution
}

\author{
Kentarou Tanabe* and Shigehiro Nagatak $\oplus^{*}$ \\ Yukawa Institute for Theoretical Physics, Kyoto University, \\ Oiwake-cho Kitashirakawa Sakyo-ku, Kyoto 606-8502, Japan
}

(Dated: December 2, 2018)

\begin{abstract}
Blandford-Znajek mechanism, by which the rotational energy of a black hole is extracted through electromagnetic fields, is one of the promising candidates as an essential process of the central engine of active compact objects such as Gamma-Ray Bursts. The only known analytical solution of this mechanism is the perturbative monopole solution for Kerr parameter $a$ up to the second order terms. In order to apply Blandford-Znajek mechanism to rapidly rotating black holes, we try to obtain the perturbation solution up to the fourth order. As a result, we find that the fourth order terms of the vector potential diverge at infinity, which implies that the perturbation approach breaks down at large distance from the black hole. Although there are some uncertainties about the solution due to the unknown boundary condition at infinity for the fourth order terms, we can derive the evaluation of the total energy flux extracted from the black hole up to fourth order of $a$ without any ambiguity. Further more, from the comparison between the numerical solution that is valid for $0<a<1$ and the fourth order solution, we find that the fourth order solution reproduces the numerical result better than the second order solution. At the same time, since the fourth order solution does not match well with numerical result at large $a$, we conclude that more higher order terms are required to reproduce the numerical result.
\end{abstract}

PACS numbers: 04.70.-s,95.30.Qd,95.30.Sf,97.60.Lf

\section{INTRODUCTION}

Gamma ray bursts(GRBs) are the most powerful explosions in the Universe [1, 2]. GRBs release $10^{51}-$ $10^{53} \mathrm{erg}$ in a few seconds, which corresponds to a rest mass energy of the sun. The mechanism of the central engine of GRBs is not still understood well. However, from the fact that some GRBs are associated with supernova explosions [3, 4], we can guess that there might be a black hole at the center of a GRB and its energy source may be the rotation energy of the black hole. In order to extract the rotational energy of the black hole, the Blandford-Znajek mechanism was proposed [5, [6, 7]. In this mechanism, the rotational energy is extracted in a form of the Poynting flux that is realized by the surrounding electromagnetic fields. The scale of extracted energy from the black holes is, in a rough estimate [8],

$$
\begin{aligned}
\dot{E} & \sim B^{2} \Omega_{H}^{2} M^{2} \\
& \sim 10^{51} \mathrm{erg} / \mathrm{s}\left(\frac{a}{M}\right)^{2}\left(\frac{M}{10 M_{\odot}}\right)^{2}\left(\frac{B}{10^{15} \mathrm{G}}\right)^{2}
\end{aligned}
$$

where $B$ is a magnetic field, $M$ is the mass of the black hole, $a$ is the specific angular momentum of black hole (Kerr parameter). Compared with the energy scale of GRBs, this energy scale seems to be a good one.

Although Blandford and Znajek (1977) obtained an analytical solution for this mechanism by assuming the shape of the magnetic field (monopole magnetic field), this solution has two points that should be investigated

\footnotetext{
*Electronic address: tanabe@yukawa.kyoto-u.ac.jp

${ }^{\dagger}$ Electronic address: nagataki@yukawa.kyoto-u.ac.jp
}

further. First, this solution is the perturbative solution for Kerr parameter $a$. Thus, this solution is valid only for a slowly rotating black hole $(a \ll M)$. Second, it is very difficult to impose proper boundary conditions. The equation we have to solve is the second order differential equation, thus we must impose two boundary conditions for a solution. In the original paper, assuming force free condition in all region, they imposed boundary conditions such that at event horizon the magnetic field is finite and at infinity the solution should connect with another force free solution obtained in Newton gravity 9]. The boundary condition at event horizon is plausible [8], but since at large $r$, there is no guarantee that we can use force free condition, we have to be careful for the boundary condition at infinity.

The aim of this paper is to evaluate more accurately the energy flux extracted from a rotating black hole by the Blandford-Znajek mechanism, i.e. to derive the fourth order terms of Kerr parameters for the monopole solution. Although there is two natural boundary conditions for the second order solution, proper boundary conditions are not known for the higher order solutions. Thus, when we solve the field equations, we impose a boundary condition only at the event horizon while we do not impose any boundary condition at large $r$ in this study, and we investigate the behavior of the magnetic field at large $r$. In particular, we examine whether we can impose the force free condition at infinity in order to check the validity of the force free boundary condition. Further, we compare the analytical solution we obtain in this study with the numerical calculation in order to evaluate the contribution of the fourth order terms to the extracted energy flux from the black hole.

The plan of this paper is as follows. In section 2, we solve the perturbation equations for Kerr parameter $a$ 
by imposing a boundary condition at the event horizon and estimate the total energy flux extracted from a rotating black hole. Here, we use Kerr-Schild coordinate since this coordinate is regular at the event horizon. Although the second order solution is shown to be regular in a hole region, we show the fourth order solution diverges irrespective of any boundary condition at infinity, which means the perturbation approach breaks down at large distance from the black hole. Our notation follows McKinney and Gammie [6] in section 2. In section 3, we compare the analytical solution obtained in section 2 with numerical solution which is valid for $0<a<M$. In section 4 , we summarize and discuss our results.

\section{METHOD OF CALCULATION}

As stated in section 1, in Blandford-Znajek mechanism, rotational energy of a black hole is extracted through electromagnetic fields and this extracted energy may be the energy source of GRBs. When the energy is extracted through electromagnetic fields, it propagates in the form of the Poynting flux vector. Here, we give the formula of Poynting flux vector in Kerr space-time for arbitrary shape of magnetic fields. As mentioned above, we use Kerr-Schild coordinate here for convenience:

$$
\begin{aligned}
d s^{2} & =\left(-1+\frac{2 r}{\Sigma}\right) d t^{2}+\left(1+\frac{2 r}{\Sigma}\right) d r^{2}+\Sigma d \theta^{2} \\
& +\frac{\sin ^{2} \theta}{\Sigma}\left(\left(r^{2}+a^{2}\right)^{2}-\triangle a^{2} \sin ^{2} \theta\right) d \phi^{2}+\frac{4 r}{\Sigma} d t d r \\
& -\frac{4 a r \sin ^{2} \theta}{\Sigma} d t d \phi,-2 a \sin ^{2} \theta\left(1+\frac{2 r}{\Sigma}\right) d r d \phi
\end{aligned}
$$

where

$$
\begin{aligned}
& \Sigma=r^{2}+a^{2} \cos ^{2} \theta \\
& \triangle=r^{2}-2 r+a^{2}
\end{aligned}
$$

and we use the unit in which $G=c=$ $M$ (black hole mass $)=1$. The energy momentum tensor of electromagnetic fields is written in terms of $F_{\mu \nu}=$ $\partial_{\mu} A_{\nu}-\partial_{\nu} A_{\mu}$

$$
T_{\mu \nu}^{E M}=F_{\mu \rho} F_{\nu}{ }^{\rho}-\frac{1}{4} F_{\alpha \beta} F^{\alpha \beta} g_{\mu \nu}
$$

$-T_{t}^{r}$ component of $T_{\nu}^{\mu}$ is the $r$ component of Poynting flux vector.

We can expect that matter's contribution to the total energy can be ignored near the black hole, so force free condition is valid [5]. Force free condition is represented by electromagnetic tensor $F_{\mu \nu}$ as follows:

$$
F_{\mu \nu} J^{\nu}=0
$$

where $J^{\mu}$ is the current vector. This approximation means that the inertial force by matter is ignored and the total energy momentum tensor is composed of electromagnetic one only:

$$
T_{\text {total }}^{\mu \nu}=T_{\text {matter }}^{\mu \nu}+T_{E M}^{\mu \nu} \cong T_{E M}^{\mu \nu}
$$

In other word, matter energy is ignorable compared with electromagnetic energy. Thus, in the force free region, equation of motion of electromagnetic field becomes as follows:

$$
\nabla_{\mu} T_{E M}^{\mu \nu}=0
$$

We use $T^{\mu \nu}$ as $T_{E M}^{\mu \nu}$ below.

Generally, the number of independent variables is six; electric fields and magnetic fields. However, when we impose the force free condition, there are four independent variables. Further, assuming the the force free condition (6), stationarity, and axisymmetry, there is a relation among vector potential terms $A_{\mu}$ :

$$
A_{\phi, \theta} A_{t, r}-A_{t, \theta} A_{\phi, r}=0
$$

which makes the number of independent variables three. This relation implies that part of the electric field components are represented by magnetic field components. Also, we can define the angular velocity $\Omega_{F}$ of electromagnetic fields from (9):

$$
\frac{A_{t, \theta}}{A_{\phi, \theta}}=\frac{A_{t, r}}{A_{\phi, r}} \equiv \Omega_{F} .
$$

All components of the electric fields are represented by this angular velocity $\Omega_{F}$ and magnetic field components $B^{i}(i=r, \theta, \phi)$. After all, independent variables are $\phi$ component of magnetic field $B^{\phi}, \phi$ component of vector potential $A_{\phi}$ and angular velocity of electromagnetic field $\Omega_{F}$. Here, we define magnetic field components as follows:

$$
\begin{aligned}
B^{r} & =\frac{1}{\sqrt{-g}} F_{\theta \phi}, \\
B^{\theta} & =\frac{1}{\sqrt{-g}} F_{\phi r}, \\
B^{\phi} & =\frac{1}{\sqrt{-g}} F_{r \phi} .
\end{aligned}
$$

Using these variables, we can evaluate the energy flux $-T_{t}^{r}$ of electromagnetic field as

$-T_{t}^{r}=-2\left(B^{r}\right)^{2} \Omega_{F}\left(\Omega_{F}-\frac{a}{2 r}\right) \sin ^{2} \theta-B^{r} B^{\phi} \Omega_{F} \triangle \sin ^{2} \theta$.

On the event horizon $\left(r=r_{+} \Rightarrow \triangle=0\right)$, this formula becomes

$$
-T_{t}^{r}=2\left(B^{r}\right)^{2} \Omega_{F} r_{+}\left(\Omega_{H}-\Omega_{F}\right)
$$

where $\Omega_{H}$ is the angular velocity of the black hole. Thus rotational energy can be extracted through electromagnetic fields in the force free region if and only if $0<\Omega_{F}<\Omega_{H}$. 
What we want to evaluate is the total energy flux of the extracted energy from the black hole. Its total energy flux $\dot{E}$ is represented as the integration of the energy flux at $r=$ const. surface.

$$
\dot{E}=-2 \pi \int_{0}^{\pi} \sqrt{-g} T_{t}^{r} d \theta
$$

From stationarity and axisymmetry of Kerr space time, this total energy flux $\dot{E}$ is independent of $r$ at which the integration is operated.

The formula (16) which we derive here can be used for any shape of magnetic fields. In this section we evaluate this total energy flux for the monopole magnetic field. Since it is very difficult to solve the equation of motion (8) analytically, we treat Kerr metric as the perturbation in Kerr parameter from Schwarzschild metric to obtain analytical solutions.

\section{A. second order terms}

Here, we explain the monopole solution which Blandford and Znajek obtained by perturbation method for Kerr parameter $a$. At first, the unperturbed solution is the monopole solution in Schwarzschild space time. This unperturbed solution satisfies

$$
\nabla_{\mu}^{(0)} T_{\nu}^{\mu}=0
$$

where (0) means that Schwarzschild metric is used. The first order monopole solution is

$$
\begin{gathered}
A_{\phi}^{(0)}=-C \cos \theta, \\
B^{\phi(0)}=0, \\
\Omega_{F}^{(0)}=0
\end{gathered}
$$

where $C$, strength of the magnetic field, is the constant. In this solution, since electromagnetic fields do not rotate, rotational energy of black hole cannot be extracted.

In order to obtain the monopole solution around a slowly rotating black hole, we consider perturbation for Kerr parameter $a$. Taking account of axisymmetry, we expand the solution up to the second order as follows:

$$
\begin{gathered}
A_{\phi}=-C \cos \theta+a^{2} A_{\phi}^{(2)}, \\
B^{\phi}=a B^{\phi(1)} \\
\Omega_{F}=a \omega^{(1)} .
\end{gathered}
$$

We also use the expanded Kerr-Schild metric up to the second order in $a$.

The equation we solve is $\nabla_{\mu} T_{\nu}^{\mu}=0$. The $t, \phi$ components of this equation represent the conservation equation for energy and angular momentum of the electromagnetic fields, respectively. The $r, \theta$ components are the trans- field equations. Here, we impose boundary conditions.

$$
\begin{gathered}
\left.A_{\phi}^{(2)}\right|_{\text {on the event horizon }}=\text { finite }, \\
A_{\phi}^{(2)} \rightarrow O\left(\frac{1}{r}\right)(r \rightarrow \infty), \\
\left.B^{\phi(1)}\right|_{\text {on the event horizon }}=\text { finite. }
\end{gathered}
$$

The conditions (24), (26) in Kerr-Schild coordinate corresponds to the condition in Boyer-Lindquist coordinate such that the magnetic field that FIDO (fiducial observer) feels should be finite [10], and (25) is required to connect the solution to the Michel's solution. In ref [6], regularity at infinity and separability of $A_{\phi}^{(2)}$ are taken as the boundary conditions. Although the boundary conditions are different from ours, the result we obtain below is the same with McKinney and Gammie [6]. As we see in fourth order calculation, $A_{\phi}^{(4)}$ is not separable. Thus we do not adopt the separability as a boundary condition in this study.

Under these boundary conditions, the solutions are derived as follows:

$$
\begin{gathered}
A_{\phi}^{(2)}=C f(r) \cos \theta \sin ^{2} \theta, \\
B^{\phi(1)}=-\frac{C}{4 r^{2}}\left(\frac{1}{2}+\frac{2}{r}\right), \\
\omega^{(1)}=\frac{1}{8}
\end{gathered}
$$

where

$$
\begin{aligned}
f(r)= & \left(L i_{2}\left(\frac{2}{r}\right)-\ln \left(1-\frac{2}{r}\right) \ln \left(\frac{r}{2}\right)\right) \frac{r^{2}(2 r-3)}{8} \\
& +\frac{1+3 r-6 r^{2}}{12} \ln \left(\frac{r}{2}\right)+\frac{11}{72}+\frac{1}{3 r}+\frac{r}{2}-\frac{r^{2}}{2}
\end{aligned}
$$

and

$$
L i_{2}(x)=-\int_{0}^{1} \frac{\ln (1-t x)}{t} d t .
$$

By choosing $\omega^{(1)}=1 / 8$, we can use the force free condition at large $r$ consistently. We evaluate the total energy flux of the electromagnetic field.

$$
\begin{aligned}
\dot{E} & =-2 \pi \int_{0}^{\pi} \sqrt{-g} T_{t}^{r} d \theta \\
& =\frac{\pi}{24} a^{2} C^{2} .
\end{aligned}
$$

Eq. (32) is the result that Blandford and Znajek (1977) obtained.

\section{B. fourth order terms}

Here, we try to obtain the fourth order terms for Kerr parameter. Although the method to derive the fourth order terms is basically same as the second order case, 
we have a problem with the boundary condition as mentioned in section 1 . We must impose two boundary conditions. One is the condition at the event horizon. This is same as the second order solution. As for another boundary condition, we cannot guess anything since we do not know where the force free condition breaks down and which solution we can connect to. That is why we impose a boundary condition only at the event horizon and we solve the fourth order equations. We also examine whether we can put a force free boundary condition at large $r$ using the derived fourth order solution.

The equation we solve is the fourth order one for Kerr parameter $a$ of $\nabla_{\mu} T_{\nu}^{\mu}=0$. We expand the variables as follows:

$$
\begin{gathered}
A_{\phi}=-C \cos \theta+a^{2} A_{\phi}^{(2)}+a^{4} A_{\phi}^{(4)}, \\
B^{\phi}=a B^{\phi(1)}+a^{3} B^{\phi(3)}, \\
\Omega_{F}=a \omega^{(1)}+a^{3} \omega^{(3)} .
\end{gathered}
$$

By checking the fourth order terms of the $t$ and $\theta$ for the equation $\nabla_{\mu} T_{\nu}^{\mu}=0$, one can guess that the $\theta$ dependence should be as follows:

$$
\begin{gathered}
A_{\phi}^{(4)}=h_{1}(r) \cos \theta+h_{3}(r) \cos ^{3} \theta+h_{5}(r) \cos ^{5} \theta, \\
B^{\phi(3)}=g_{0}(r)+g_{2}(r) \cos 2 \theta, \\
\omega^{(3)}=b_{0}+b_{2} \cos 2 \theta
\end{gathered}
$$

where $b_{0}, b_{2}$ are constants. When we impose boundary condition at the event horizon like the second order solution, we can solve the $\phi$ component of the equation for $g_{0}, g_{2}$. The solutions are as follows:

$$
\begin{aligned}
g_{0}(r)= & \frac{1}{576 r^{5}(r-2)}\left(-288+456 r+92 r^{2}+109 r^{3}+36 r^{6}-1152 b_{0} r^{3}+\frac{r^{4}}{2}\left(6 \pi^{2}-521+1152 b_{0}\right)\right. \\
& \left.+3 r^{2}\left(8+22 r-56 r^{2}+6 r^{3}+12 r^{4}\right)+9 r^{4}\left(12-11 r-r^{2}+2 r^{3}\right)\left(\ln \left(\frac{r-2}{r}\right) \ln \left(\frac{r}{2}\right)-L i_{2}\left(\frac{2}{r}\right)\right)\right) \\
g_{2}(r)= & \frac{1}{192 r^{5}(r-2)}\left(-96+120 r+20 r^{2}+125 r^{3}+24 r^{5}+12 r^{6}-384 b_{2} r^{3}+\frac{r^{4}}{2}\left(384 b_{2}-95-2 \pi^{2}\right)\right. \\
& \left.+r^{2}\left(24+66 r-164 r^{2}+30 r^{3}+12 r^{4}\right)+3 r^{4}\left(36-33 r+3 r^{2}+2 r^{3}\right)\left(\ln \left(\frac{r-2}{r}\right) \ln \left(\frac{r}{2}\right)-L i_{2}\left(\frac{2}{r}\right)\right)\right) .
\end{aligned}
$$

The behavior of these solutions at large $r$ are

$$
\begin{aligned}
& g_{0}(r)=\frac{1152 b_{0}+6 \pi^{2}-139}{1152 r^{2}}+O\left(\frac{1}{r^{3}}\right), \\
& g_{2}(r)=\frac{1152 b_{2}-6 \pi^{2}+67}{1152 r^{2}}+O\left(\frac{1}{r^{3}}\right) .
\end{aligned}
$$

These solutions depend on the constants in the angular velocity of the electromagnetic fields $b_{0}, b_{2}$, which must be determined by another boundary condition that we do not know. The total energy flux of electromagnetic field is

$$
\begin{aligned}
\dot{E} & =-2 \pi \int_{0}^{2 \pi} \sqrt{-g} T_{t}^{r} d \theta \\
& =\frac{\pi}{24} a^{2} C^{2}+\frac{\pi\left(56-3 \pi^{2}\right)}{1080} a^{4} C^{2} .
\end{aligned}
$$

This is the value we want to evaluate. Fortunately, this result does not depend on the undetermined constants $b_{0}, b_{2}$. Probably, it is by chance. On the other hand, at large $r$, we can solve the $r$ component of the fourth order equation of $\nabla_{\mu} T_{\nu}^{\mu}=0$ for $A_{\phi}^{(4)}$. The behavior is

$$
A_{\phi}^{(4)} \rightarrow O\left(r^{2}\right)
$$

No matter how we choose $b_{0}, b_{2}$ which must be determined by a boundary condition, we cannot make $A_{\phi}^{(4)}$ $O(1 / r)$ as the second solution at large $r$. This result implies that this solution cannot work well at large $r$ because perturbation method breaks down there. However, the evaluation of the total energy flux is valid because, as mentioned above, $\dot{E}$ is independent of $r$ whether we use perturbation method or not.

\section{COMPARISON WITH NUMERICAL CALCULATION}

In order to see how well the forth-order term works to describe the total energy flux for large Kerr parameter, we have performed numerical simulations of the monopole solution and compared the results with the analytical, second-order and forth-order solutions. 
We have developed a two-dimensional General Relativistic Magneto-Hydro Dynamics (GRMHD) code following 11 12]. We have adopted a conservative, shockcapturing scheme with Harten, Lax, and van Leer (HLL) flux term [13] with flux-interpolated constrained transport technique [14]. We use a third-order TVD RungeKutta method for evolution in time, while Monotonized central slope-limited linear interpolation method is used for second-order accuracy in space [15]. 2D scheme (2dimensional Newton-Raphson method) is adopted for transforming between conserved variables and primitive variables [12]. We used a simple gamma-law equation of state with $\gamma=4 / 3$. Modified Kerr-Schild coordinate is adopted with mass of the black hole $(M)$ fixed where the Kerr-Schild radius $r$ is replaced by the logarithmic radial coordinate $x_{1}=\ln r$. In the following, we use $G=M=c=1$ unit.

The computational domain is axisymmetric, with a grid that extends from $r_{\text {in }}=0.98 r_{+}$to $r_{\text {out }}=230$ and from $\theta=0$ to $\theta=\pi$ where $r_{+}$is the outer event horizon. The numerical resolution is $300 \times 300$. As an initial condition, we put the 0th order terms of the monopole solution around the black hole [16]. That is, $\Re^{\mu}=-n_{\nu}^{*} F^{\mu \nu}=(0, \alpha C \sin \theta / \sqrt{-g}, 0,0)$ in the KerrSchild coordinate where $n_{\nu},{ }^{*} F^{\mu \nu}, \alpha, g$ are the normal observer's four-velocity, the dual field tensor, Lapsefunction, and determinant of the Kerr-Schild metric. The numerical constant $C$ is set to be unity in this study. The plasma velocity relative to the FIDO is set to zero initially, and its pressure and density are set to the same value of $P=\rho=\Re^{2} / 100$ so that force free approximation is a good one. Also, to keep the magnetization reasonably low, when the critical condition $0.01 \Re^{2} \geq \Gamma^{2} \rho+\left(\gamma \Gamma^{2}-(\gamma-1)\right) U$ is satisfied, density and internal energy $U$ is increased by the same factor so that the critical condition holds [16]. Here $\Gamma$ is the bulk Lorentz factor of the fluid measured in the Kerr-Schild coordinate. We have performed numerical simulations with the Kerr parameters $0,0.01,0.05,0.1,0.15,0.2$, $0.3,0.4,0.5,0.6,0.7,0.8,0.9,0.95,0.99$, and 0.995 until time $\mathrm{T}=200$.

In Fig. 1 we plot the total energy flux at the final stage for small Kerr parameter $(0 \leq a \leq 0.2)$ by rectangular points. The total energy flux was evaluated at $r=20$, although we found that the total energy flux is insensitive to the radius where it is evaluated. This means that the conservation of the total energy flux has been confirmed numerically. Dashed line is just the interpolation of the calculated values. For comparison, the second-order analytical solution is shown by the dotted line and the forthorder analytical solution is shown by the solid line. From this comparison, we can see that all of them coincide with each other that means the Blandford-Znajek solution is a really good approximation for small Kerr parameters.

The situation becomes different drastically for large Kerr parameter. In Fig. 2, we plot the same values with Fig. 1, but with wide range of the Kerr parameter $(0 \leq$ $a \leq 1)$. We can see clearly the difference among three

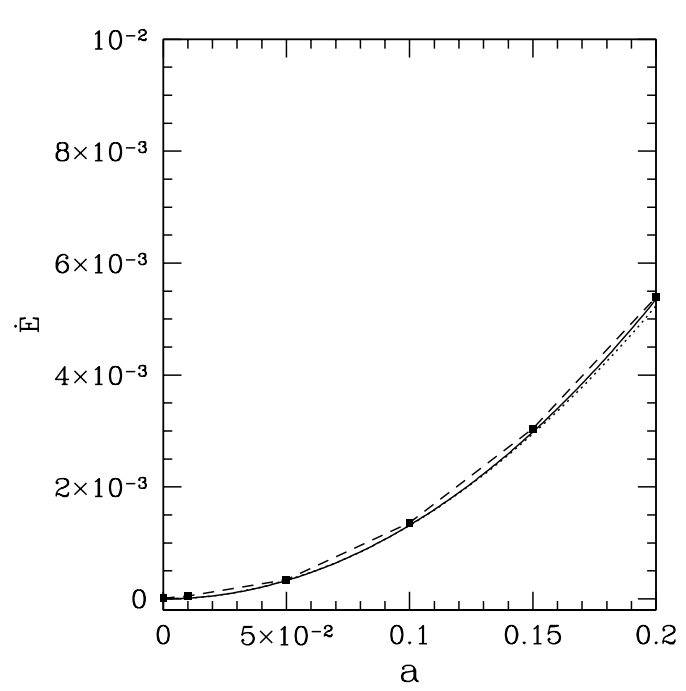

FIG. 1: Comparison of the derived, conserved, total energy flux. Dashed line with rectangular points is numerical result for small Kerr parameter $(0 \leq a \leq 0.2)$, dotted line shows the second-order analytical solution, and solid line represents the forth-order analytical solution.

cases. However, we would like to emphasize the following point: we could confirm that the forth order analytical solution is a better approximation rather than the second order analytical solution. In fact, the deviation between the numerical result and forth order analytical solution is less than that between the numerical result and second order analytical solution. Of course, we could also see that much higher-order analytical solution is required to reproduce the numerical result, which is out of the scope in this study.

\section{DISCUSSION AND CONCLUSION}

\section{A. discussion}

We have tried to solve the fourth order terms for the Blandford-Znajek monopole solution to evaluate extracted energy flux more accurately. Since the equation for $B^{\phi}$ is a first order differential equation, we could solve by imposing a boundary condition at the horizon. However, $A_{\phi}$ obeys the second order differential equation, thus we need to put two boundary conditions. Blandford and Znajek (1977) imposed the boundary conditions at the horizon and infinity. The boundary condition at infinity was chosen to connect the Michel's solution. In this study, we tried to impose the same boundary conditions for the fourth order solution. However, we found that the fourth order solution can not be connected to 


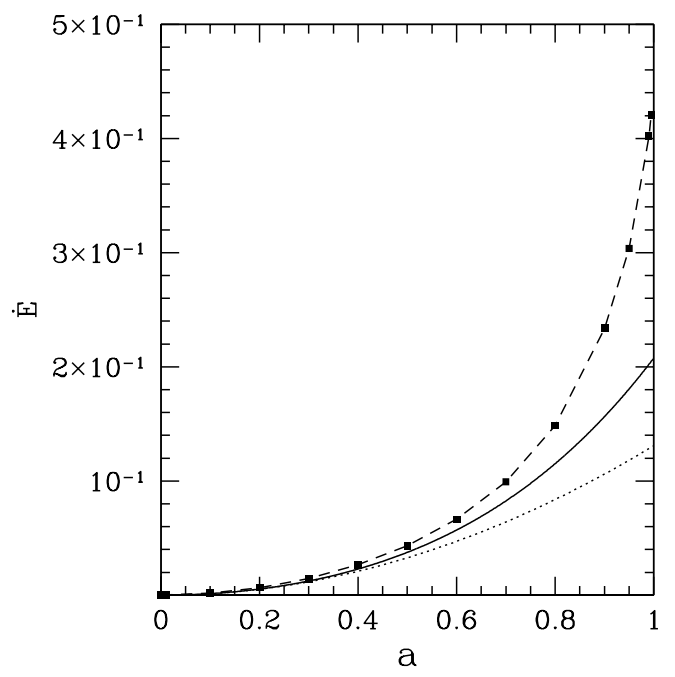

FIG. 2: Same with Fig.1, but for wide range of the Kerr parameter $(0 \leq a \leq 1)$

Michel's solution with any boundary condition at infinity. After all, we could not find the fourth order solution of $A_{\phi}$ assuming the force free condition. Furthermore, we found the perturbation method for Kerr parameter $a$ breaks down at large $r$ because of the behavior $A_{\phi}^{(4)} \sim r^{2}$. Fortunately, the fourth order term of the total energy flux extracted from a rotating black hole, which we want to evaluate most, does not depend on the solution we could not solve.

The boundary condition which we could not specify was considered in [17, 18]. In general, the equation for the stream function $A_{\phi}$ which is called Grad-Shafranov equation has some singular surfaces [19, 20]. In the force free limit, this equation is singular at horizon and at light cylinder. Thus, in order to determine the solution uniquely, we have to impose proper boundary conditions at horizon and light cylinder. However, boundary conditions to be imposed is not understood well [17].

We have compared the numerical result with our fourth order solution. The numerical result we use is the result in GRMHD simulation. But for the evaluation of the total energy flux, GRMHD result is almost same as the result in GRFFE (General Relativistic Force Free Electrodynamics) simulation [21], so we would get the same conclusion when we compare the GRFFE result with our fourth order solution. However, for the details of the electromagnetic fields (e.g. shape of the field lines), the numerical result of GRMHD would be different from the one in GRFFE. As our future work, we want to examine the difference among our analytical result, the numerical result of GRMHD, and the one of GRFFE.

In our calculation, we assume an infinitely thin disk, and cannot self-consistently determine a constant $C$ in Eq. (18) which determines a magnitude of the magnetic field. However, in a realistic accretion disk, $C$ may depend on $a$ 22] and total energy flux may be determined self-consistently. Thus, when Blandford-Znajek mechanism is considered with a realistic accretion disk, our result Eq.(43), which also contains the constant $C$, has to be treated carefully.

\section{B. conclusion}

Our aim of this paper is to evaluate the total energy flux extracted from a rapidly rotating black hole by Blandford-Znajek mechanism more accurately. As a result, although we could not the obtain the all perturbation solution up to the fourth order, we could evaluate the total energy flux extracted from a rotating black hole without any ambiguity irrespective of the unknown boundary condition at infinity. Also, we found the perturbation method for the fourth order terms breaks down at large $r$ by solving the equation for $A_{\phi}^{(4)}$ under the force free condition. This would be because in the monopole solutions, the force free condition can not be compatible with a rotating black hole at the fourth order of Kerr parameter.

From the comparison between the numerical solution that is valid for $0<a<1$ and the fourth order solution, we find that the fourth order solution reproduces the numerical result better than the second order solution. At the same time, since the fourth order solution does not match well with numerical result at large $a$, we conclude that more higher order terms are required to reproduce the numerical result.

\section{Acknowledgments}

We are grateful to R. Blandford, J. McKinney, and K. Ruben for useful discussion. We also appreciate K. Murase and J. Aoi for important discussions and comments. The computation was carried out on NEC SX-8 and SGI Altix3700 BX2 at Yukawa Institute for Theoretical Physics, Kyoto University. This work is in part supported by a Grant-in-Aid for the 21st Century COE "Center for Diversity and Universality in Physics" from the Ministry of Education, Culture, Sports, Science and Technology of Japan. S.N. is partially supported by Grants-in-Aid for Scientific Research from the Ministry of Education, Culture, Sports, Science and Technology of Japan through No. 19047004, 19104006, and 19740139. 
[1] Meszaros, P., Rep. Prog. Phys., 69, 2259 (2006)

[2] Piran, T., Rev. Mod. Phys. 76, 1143 (2005)

[3] Galama, T.J., et al., Nature, 395, 670 (1998)

[4] Hjorth, J., et al., Nature, 423, 847 (2003)

[5] Blandford, R.D., Znajek, R.L., Mon. Not. R. Astron. Soc., 179, 433 (1977)

[6] McKinney, J.C., Gammie, C.F., Astrophys. J 611, 977 (2004)

[7] Lee, H.K., Wijers, R.A.M.J., Brown, G.E., Phys.Rep. 325,83 (2000)

[8] Thorne, K.S., Price, R.H. and Macdonald, D.A., Black Holes: The Membrane Paradgim (1986)

[9] Michel, F.C., Astrophys. J. Lett. 180, L133 (1973)

[10] Znajek, R.L., Mon. Not. R. Astron. Soc., 179, 457 (1977)

[11] Gammie, C.F., McKinney, J.C., Tóth, G., Astrophys. J 589, 444 (2003)

[12] Noble, S.C., Gammie, C.F., McKinney, J.C., Del Zanna,
L., Astrophys. J 641, 626 (2006)

[13] Harten, A., Lax, P.D., van Lerr, B., SIAM Rev. 25, 35 (1983)

[14] Tóth, J. Compt. Phys. 161, 605 (2000)

[15] van Leer, B.J., J. Compt. Phys. 23, 276 (1977)

[16] Komissarov, S.S., Mon. Not. R. Astron. Soc. 350, 1431 (2004)

[17] Uzdensky, D.A., Astrophys. J. 603, 652 (2004)

[18] Uzdensky, D.A., preprint arXiv:astro-ph/0410715

[19] Beskin, V.S., preprint arXiv:astro-ph/0409076

[20] Kim, H., Lee, H.M., Lee, C.H. and Lee, H.K., Mon. Not. R. Astron. Soc. 358, 998 (2005)

[21] Blandford, R.D., Ruben, K., private communications (2007)

[22] McKinney, J.C., Astrophys. J. 630 L5 (2005) 